\title{
Hippocampal abnormalities and sudden childhood death
}

\author{
Jeffrey Noebels ${ }^{1}$ \\ ${ }^{1}$ Department of Neurology, Baylor College of Medicine, One, Baylor Plaza, Houston, TX 77030, \\ USA
}

\begin{abstract}
Do postmortem structural changes in the hippocampal formation explain the still mysterious mechanism of premature mortality in otherwise normal children? Could clinically unrecognized seizures provide a missing link between two syndromes featuring sudden unexpected death in children, SUDC, and individuals with a history of epilepsy, SUDEP?
\end{abstract}

Two recent reports [1,2] highlight unexpected patterns of epileptiform pathology in the forebrain of infantile SUDC cases. The novelty involves the region where the structural changes were discovered, and their striking overlap with those described in pediatric febrile seizure cohorts that often evolve into hippocampal sclerosis and temporal lobe epilepsy [3]. In a pilot forensic study of 153 SUDC cases, the three most common morphological features identified were focal dentate granule cell bilamination, asymmetry, and malrotation, seen in $47.8 \%$ (33/69) of SUDC cases compared to $13.3 \%$ (3/23) of explained cases. Nearly half (59/121) of the cases had a personal or family history of febrile seizures, and a fever around the time of death. A further compelling similarity was that $93 \%$ of the victims were found prone during a sleep period; SUDEP shows a similar nocturnal and positional predisposition. Exome testing found 1 of 3 genotyped cases was positive for a de novo KVLQT1 cardiac LQT mutation, a proven cause of SUDEP [4]. Such mutations of ion channel genes coexpressed in heart and brain are a recent and important risk factor for SUDEP, where they cause seizures, arrhythmias, and premature death [4]. Interestingly, SUDEP genes lead to hippocampal and dentate gyrus abnormalities in mouse models, and seizures themselves are a well established cause of further structural deformation in immature brain. Therefore the hippocampal lesions represent a newly forming seizure focus with a high probability of progression to epilepsy, warranting a new term, 'epilepsy in situ'.

Since the hippocampus lies far from the lower brainstem where the forensic SIDS lamppost has shone for several decades, what makes this lesion lethal? Long focused on pontine and medullary centers where microcircuits regulate cardiorespiratory pacemaking, the search for causative abnormalities has moved to higher levels of central autonomic pathways mediating arousal and auto resuscitation [5]. Forebrain control over cardiorespiratory nuclei resides in the rostral limbic system, including amygdalo-hippocampal circuitry and insula, loci well known for their low focal seizure thresholds. Hyperexcitability and seizures confined to these circuits may be clinically subtle, and even in the absence of a seizure, SUDEP genes lower the threshold for brainstem spreading depression, a lethal event [6]. 
Ultimately, sudden death risk depends upon a diverse combination of structural, physiological, and genetic features. The etiology in an individual case may be unclear, and whether inherited or acquired, how early the biological risk begins (in utero, perinatal, or thereafter) will vary. Given the spectrum of anatomical pathways and excitability molecules that promote their normal connectivity, the risk of sudden unexpected death depends upon the collective status of scores of causal gene variants that unleash autonomic instability and seizures; such 'pre-epilepsy' cases may present either with or without structural pathology or clinically manifest seizure activity.

Indeed, as the authors point out, the visible lesion they define as 'hippocampal malformation associated with sudden death' (HMASD), was present in almost $50 \%$ of the total cases of SUDC, leaving the remaining $50 \%$ unexplained. In both groups, cardiac LQT gene testing deserves to become an integral element of the forensic workup. Going forward, special stains for activity-dependent cell markers that seizures leave in their wake [7] could provide further evidence to establish a forensic diagnosis of "epilepsy in situ", a new term that may be usefully applied to a microscopic epileptiform lesion with or without evidence of actual seizures.

\section{References}

1. Hefti MM, Kinney HC, Cryan JB, Haas EA, Chadwick AE, Crandall LA, et al. Sudden unexpected death in early childhood: general observations in a series of 151 cases: part 1 of the investigations of the San Diego SUDC Research Project. Forensic Sci Med Pathol. 2016; 12:4-13. [PubMed: 26782961]

2. Hefti MM, Cryan JB, Haas EA, Chadwick AE, Crandall LA, Trachtenberg FL, et al. Hippocampal malformation associated with sudden death in early childhood: a neuropathologic study: part 2 of the investigations of The San Diego SUDC Research Project. Forensic Sci Med Pathol. 2016; 12:14-25. [PubMed: 26782962]

3. Chan S, Bello JA, Shinnar S, Hesdorffer DC, Lewis DV, MacFall J, et al. Hippocampal malrotation is associated with prolonged febrile seizures: results of the FEBSTAT study. AJR. 2015; 205:106874. [PubMed: 26496555]

4. Goldman AM, Glasscock E, Yoo J, Chen TT, Klassen TL, Noebels JL. Arrhythmia in heart and brain: KCNQ1 mutations link epilepsy and sudden unexplained death. Sci Transl Med. 2009; $1: 2 \mathrm{ra6}$.

5. Wandschneider B, Koepp M, Scott C, Micallef C, Balestrini S, Sisodiya SM, et al. Structural imaging biomarkers of sudden unexpected death in epilepsy. Brain. 2015; 138:2907-19. [PubMed: 26264515]

6. Aiba I, Noebels JL. Spreading depolarization in the brainstem mediates sudden cardiorespiratory arrest in mouse SUDEP models. Sci Transl Med. 2015; 7:282ra46.

7. Houser CR, Zhang N, Peng Z, Huang CS, Cetina Y. Neuroanatomical clues to altered neuronal activity in epilepsy: from ultrastructure to signaling pathways of dentate granule cells. Epilepsia. 2012; 53(Suppl 1):67-77. 